\title{
The research on temperature sensing properties of photonic crystal fiber based on Liquid crystal filling
}

\author{
Xiangzhen Zan ${ }^{1, a}$, Liliang Qian², Miao Wang ${ }^{3,4}$ and Wenbin Liu ${ }^{5}$ \\ ${ }_{2}^{1}$ Department of Information Engineering, City College of Wenzhou University, Wenzhou city, Zhejiang province, China \\ 2 Lujiang Middle school, Hefei city, Anhui province, China \\ 3 Science and Technology on Avionics Integration Laboratory, Shanghai, China \\ ${ }^{4}$ China National Aeronautical Radio Electronics Research Institute, Shanghai, China \\ ${ }^{5}$ Department of Physics and Electronic Information Engineering, Wenzhou University, Wenzhou city, Zhejiang province, China
}

\begin{abstract}
Based on the photonic bandgap-photonic crystal fibers( PBG-PCF) fiber core fills the namitic liquid crystal. By readjusting the temperature to change the refractive index, constitute new liquid fiber-optic temperature sensor. In this paper, we use finite element COMSOL software to simulate and analyze photonic crystal optical fiber sensitive properties. The research show that after the PBG - PCF filling the liquid crystal, its mode field distribution, effective refractive index, waveguide dispersion etc changing with temperature is so big. Therefore, the properties that the refractive index of PCF mode CF changing with temperature sensitive medium, provides the theoretical basis for designing optic fiber temperature sensors.
\end{abstract}

\section{Introduction}

Photonic crystal fiber (PCF) is two dimensional photonic crystals of a kind of defects with line. This new type of optical fiber will field a new revolution in Optical fiber application domain while ordinary fiber doesn't have these characteristics. According to light's transmission mechanism, PCF can be divided into two kinds: one kind similar with the traditional optical fiber, whose refractive index of fiber core is sightly bigger than refractive index of the cladding equivalent refractive index, can form total internal reflection, that we call total internal reflective photonic crystal fibers (TIR-PCF); The other kind is photonic band gap (PBG-PCF), that uses the photonic band gap (PBG) effects of light guide. When PCF is produced, the optical transmission characteristics are determined. However, in practice, due to the fact that PCF is not very sensitive to temperature, its optical properties can be adjusted according to the requirement. Different material is filled the air hole, we can change photonic crystal optical fiber transmission characteristics and sensing properties. The refractive index of liquid crystal (LC) changing with temperature and wavelength is very sensitive. By filling the LC into photonic crystal fibers' air hole, we can adjust the temperature on the transmission properties of photonic crystal fibers. Nowadays, LC filling the cladding air holes of photonic crystal fibers is much researched, but, temperature on LC filling PCF core's research is less. This paper mainly deals with the subjects about using LC filling photonic crystal fiber's core at different temperatures, which can be adjusted photonic crystal fiber optical transmission characteristics. We use all-vector finite element

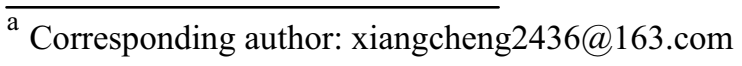

COMSOL software to simulate and analyze PCF transmission characteristic of temperature, on hexagonal structure photonic crystal core filling liquid crystal optical guidance. The main research covers temperature on the effective refractive index, mould field and the influence of chromatic dispersion waveguide.

\section{Numerical model}

Maxwell equation for the transmission characteristics of photonic crystal fiber is still applicable. We can get followed by photonic crystal fiber eigenvalue equation:

$$
\begin{aligned}
& \nabla \times\left(\frac{1}{\varepsilon_{r}} \nabla \times H\right)=\left(\frac{\omega}{c}\right)^{2} \mu_{r} H \\
& \nabla \times \nabla \times E=\left(\frac{\omega}{c}\right)^{2} \varepsilon_{r} E
\end{aligned}
$$

Where $\mathrm{H}, \mathrm{E}$ denotes the magnetic field intensity and electric field intensity respectively, $\varepsilon_{r}$ is the dielectric constant and $\mu_{r}$ is Magnetic conductivity, $\omega$ is the light wave angular frequency, $c$ is the speed of light in vacuum, $\beta$ is mode propagation constant. PBG-PCF model is constructed as shown in Figure1. 


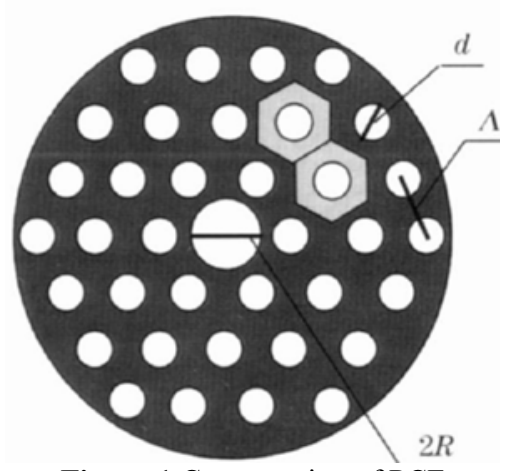

Figure 1 Cross section of PCF

Just consider the influence of temperature variations, the arrangement of liquid crystal molecules along the fiber direction is generally $\mathrm{z}$ axis direction, then $\mathrm{LC}$ in the $\mathrm{x}$ and $\mathrm{y}$ directions are ordinary polarized light, that is $n_{x}=n_{y}=n_{o}, n_{e}=n_{z}$, where $n_{o}$ and $n_{e}$ are refractive index of ordinary light and extraordinary light respectively, and function of temperature and wavelength.

Table 1

$\begin{array}{cc}\mathrm{A}=1.74 & \mathrm{~A}_{0}=1.50 \\ \mathrm{~B}=2.16 \mathrm{e}-4 & \mathrm{~B}_{0}=0.0078 \mu m^{2} \\ \beta=0.12 & C_{0}=0.0003 \mu m^{4} \\ (\Delta n)_{0}=0.6 & A_{e}=1.864 \\ T_{c}=330 K & B_{e}=0.008 \mu m^{2} \\ C_{e}=0.0026 \mu m^{4} & \\ n_{0}(T)=A-B T-\frac{(\Delta n)_{0}}{3}\left(1-\frac{T}{T_{C}}\right)^{\beta}, T<T_{C} \\ n_{e}(T)=A-B T+\frac{2(\Delta n)_{0}}{3}\left(1-\frac{T}{T_{C}}\right)^{\beta}, T<T_{C} \\ n_{0}(\lambda)=A_{0}+\frac{B_{0}}{\lambda^{2}}+\frac{C_{0}}{\lambda^{4}}, T<T_{C} \\ n_{e}(\lambda)=A_{e}+\frac{B_{e}}{\lambda^{2}}+\frac{C_{e}}{\lambda^{4}}, T<T_{C} \\ n(T, \lambda)=A-B T, T \geq T_{C}\end{array}$

\section{Mode field distribution}

Figure 2 describes the case that when wavelength of PCF is $1.55, d / \Lambda=0.5$ the mode field distribution at different temperature. We can see from the mode field distribution figure, temperature on filling the photonic crystal fiber core mode field distribution changes significantly. The reason is when fiber core filling liquid crystal, LC is sensitive to the change of temperature, in certain temperature range. Temperature increases, fiber core liquid crystal refractive index decreases, making limited decreased ability of light. So the increasing temperature makes liquid crystal filled fiber core's mode field distribution area increase. So PCF in $323 \mathrm{~K}$ mode field distribution is greater than mode field distribution in 273K . (a)

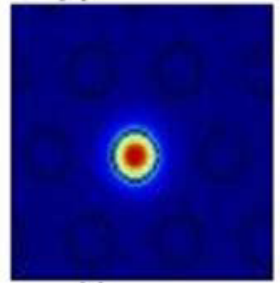

(c)

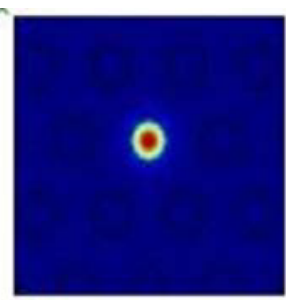

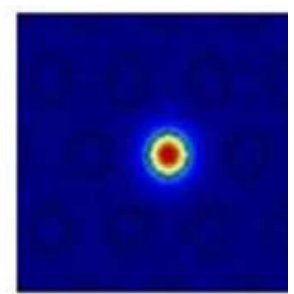

(b)

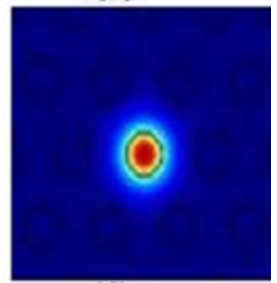

(d)
Figure 2 Mode field of PCF at $1.55 \mu \mathrm{m}$ (a) $273 \mathrm{~K}$ after filling LC, (b) $293 \mathrm{~K}$ after filling LC, (c) $313 \mathrm{~K}$ after filling LC, (d)323K after filling LC mode field distribution

\section{The influence of temperature on the effective refractive index of guide mode}

We change the PCF structure parameters: duty ratios is $0.3,0.4,0.5$ respectively, and hole spacing is $2.3 \mu \mathrm{m}$. We analyze the relationship between the effective index of guide mode with wavelength and different temperature after filling LC, with core of the PCF which is filled liquid crystal. We can see from figure 3 clearly: when the core of PCF is filled LC, the effective refractive index of the guide mode decreases with the increase wavelength. The reason is the refractive index of liquid crystal decreases with the increasing wavelength, and that long wavelength light penetrates into the cladding air holes capacity is stronger than the short-wave, for short wavelength is concentrated in the fiber core. We also find that the refractive index of the guide mode increases with increasing duty ratio, at the same hole space. Due to the larger duty ratio, the equivalent refractive index is smaller. The same ability to make light of increased restrictions guide mode, also the smaller the mode field distribution is, the effective refractive index of guide mode will be. Figure 4 is when the wavelength is $1.55 \mu \mathrm{m}$ and the PCF core is filled with LC changing with temperature, the effective index of guide mode decreases with increasing temperature. Due to the fact that liquid crystal refractive index decreases with increasing temperature, and that the core's refractive index decreases with increasing temperature, the equivalent refractive of guide mode index must decrease. In Figure 4, we still find that the refractive index of the guide mode increases with increasing duty cycle.

Figure 5 shows the relationship between the effective index and wavelength with the PCF of different temperature after filling $\mathrm{LC}$, at the duty ratio of 0.5 . In fact, from Figures 3 and 4, we know the relationship between them. Figure 5 can be further verified that the effective refractive index of the guide film decreases with 
increasing temperature, with the wavelength length increases.

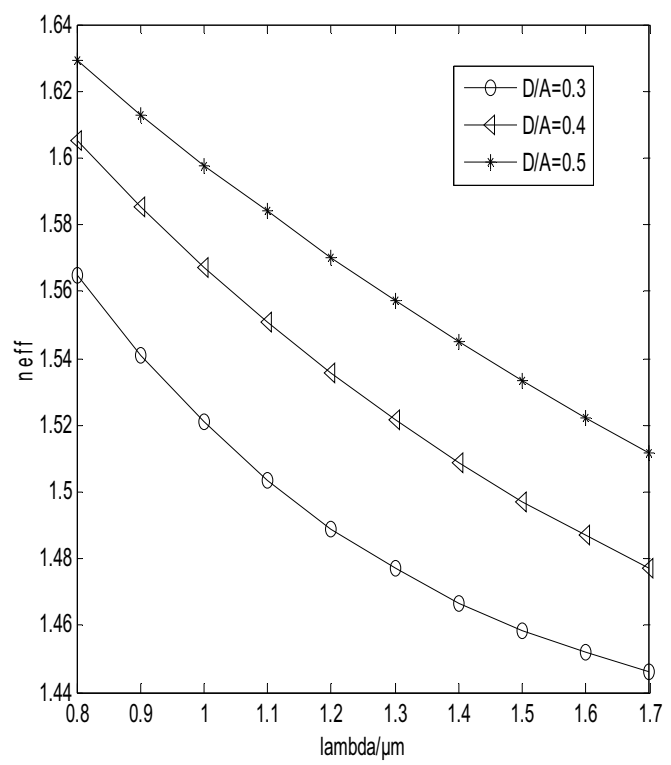

Figure 3 Relationship between the effective index and wavelength with different duty ratios of PCF after filling LC

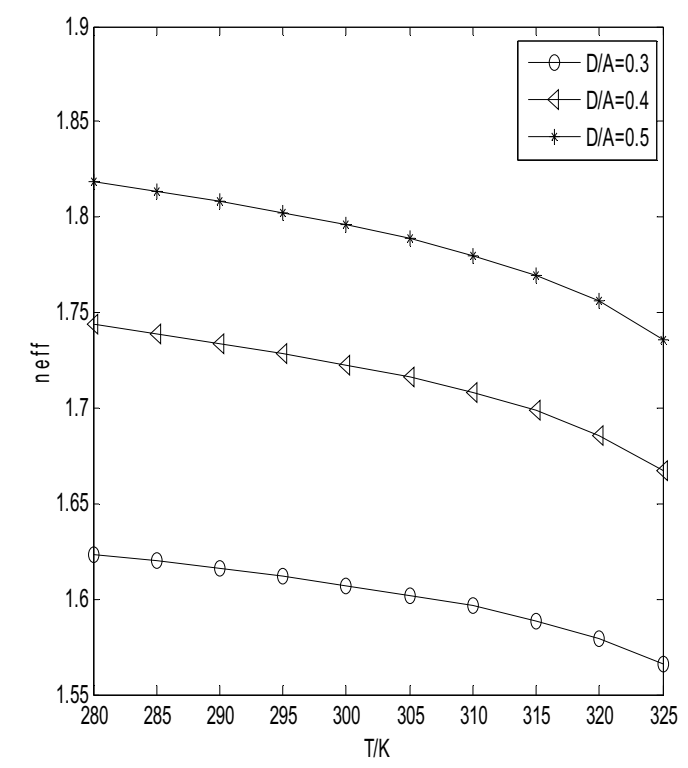

Figure 4 Relationship between the effective index and temperature with different duty ratios of PCF after filling LC

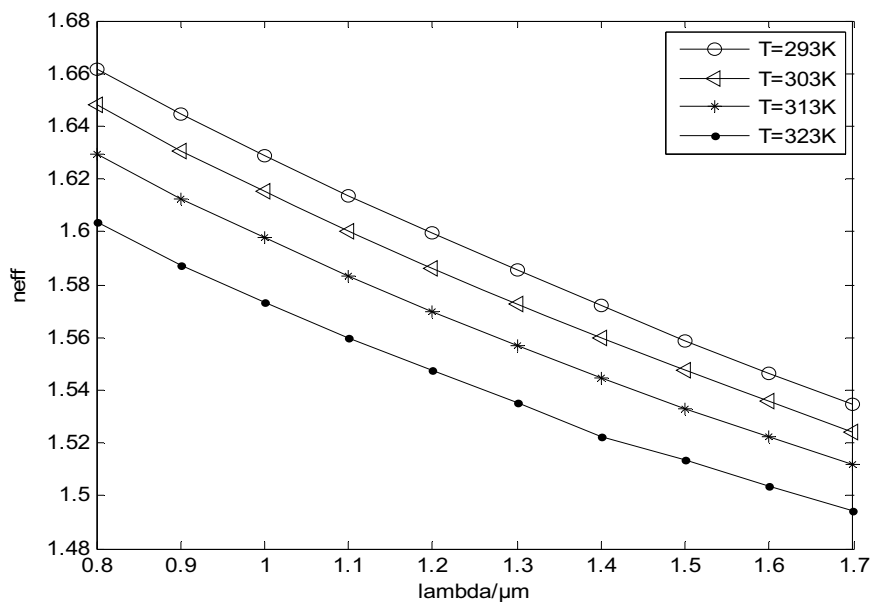

Figure 5 Relationship between the effective index and wavelength with the PCF of different temperature after filling LC

Parameters are calculated in Table 2 below.

Table 2

\begin{tabular}{lrrr}
\hline \hline $\begin{array}{l}\text { Temperatur } \\
\mathrm{e}\end{array}$ & $\mathrm{A}_{e}$ & $\mathrm{~B}_{e}\left(\mu m^{2}\right)$ & $\mathrm{C}_{e}\left(\mu m^{4}\right)$ \\
\hline $293 \mathrm{~K}$ & 1.7055 & 0.0087 & 0.0028 \\
$303 \mathrm{~K}$ & 1.6933 & 0.0078 & 0.0026 \\
& & & \\
$313 \mathrm{~K}$ & 1.6711 & 0.0091 & 0.0025 \\
$323 \mathrm{~K}$ & 1.6395 & 0.0095 & 0.0020 \\
\hline \hline
\end{tabular}

\section{The temperature characteristics of waveguide dispersion}

The PCF dispersion, includes material dispersion $\left(\mathrm{D}_{m}\right)$ and waveguide dispersion $\left(D_{w}\right)$. The different structure of the PCF, the same material dispersion, and the size is calculated by the Sellmeier formula. Waveguide dispersion is due to a pattern of the group speed of light's frequency changing with the resulting dispersion, which is defined as:

$$
\mathrm{D}_{w}=-\frac{\lambda}{c} \frac{d^{2} n_{e f f}}{d \lambda^{2}}
$$

By inputting the different wavelengths in the Comol, we can obtain the relationship between the effective refractive index of guide mode and wavelength, then six fitting in Matlab, and draw function of refractive index and wavelength, the results that are based on waveguide dispersion formula are shown in Figure 6. It can be seen that the waveguide dispersion is related to temperature and wavelength. When the wavelength is concerned, the higher temperature is, the bigger is the waveguide dispersion is, which is due to the effective refractive index of the guide film decreases with increasing 
temperature, decreasing of the refractive of guide mode waveguide dispersion increases. When the temperature is concerned, the liquid crystal waveguide dispersion increases with wavelength increasing.

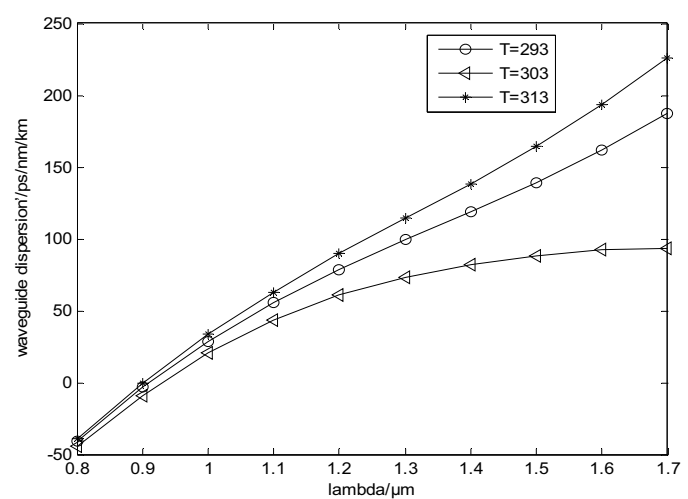

Figure 6 Relationship between the waveguide dispersion and temperature with different duty ratios of PCF after filling LC.

\section{Conclusion}

By using an all-vector finite element Comsol software, the temperature characteristic of PCF-PBG of hexagon structure arrangement was analyzed. Filling the air hole of the core with LC, the different structure as well as the relationship between the effective refractive index, mode field distribution and waveguide dispersion with temperature changing, were discussed. We find that when the core of photonic crystal fiber is filled with LC, the refractive index is very sensitive to temperature and wavelength, and that the transmission characteristic of PCF is very closely to temperature. Therefore, filling the medium of sensitive refractive index which can modulate optical fiber temperature sensing is feasible. These are valuable for design and application of temperature tunable PCF device.

\section{Acknowledgement}

This paper is supported by Aviation Foundation under Grant No.201510281326321

\section{References}

1. P.Russell. Science. 299(5605): 358-362(2003)

2. T.R.Wolinski.K.SZalliawska.S.Ertman et al. Meas. Sci.Technol. 17(5): 985-991(2006)

3. Liu Jie, Yang Changxi,C.Gu et al. Acta Optica Sinca. 26(10): 1569-1574(2006)

4. T.Kato,Y.Suetsugu,M.Nishimura. Opt.Lett, 20(22): 2279-2281(1995)

5. D.C.Zografopoulos,T.D.Tsiboukis. Opt Exp, 14(2): 914-925(2006)

6. Wen Fuzheng, Fan Wande, Li Rucheng et al.Optoelectronic Technology. 26(2): 133-137(2006)

7. W.Lin,S.Brugioni et al. Appl.Phys. 17(6): 501505(2005)

8. N.Palka. J.Phys.France, 129: 143-146(2005)
9. Qian Xiangzhong. Acta Optica Sinica. 28(5): 988991(2008)

10. Li Xuejin. Chinese Journal of Lasers. 36(6): 11401145(2009)

11. Xu, Hua, et al. Journal of Materials Chemistry C 1.38: 6087-6098(2013). 